\title{
DIAGNOSIS AND TREATMENT OF SYNCOPE
}

Michele Brignole

Heart 2007;93:130-136. doi: 10.1136/hrt.2005.080713

Take the online multiple choice questions associated with this article (see page 129) $\mathrm{n}$ the evaluation of patients with syncope, the critical first step is a detailed medical history. A diagnostic strategy based on initial evaluation is warranted. The importance of the initial evaluation goes well beyond its capability to make a diagnosis as it determines the most appropriate subsequent diagnostic pathways and risk evaluation.

\section{THE DIAGNOSTIC STRATEGY BASED ON THE INITIAL EVALUATION}

According to the Guidelines on Syncope of the European Society of Cardiology (ESC) $)^{12}$ the "initial evaluation" of a patient presenting with syncope consists of taking a careful history, and a physical examination, including orthostatic blood pressure measurements and standard electrocardiogram (ECG).

Three key questions should be addressed during the initial evaluation:

- Is loss of consciousness attributable to syncope or not? Differentiating true syncope from "nonsyncopal" conditions associated with real or apparent transient loss of consciousness is generally the first diagnostic challenge and influences the subsequent diagnostic strategy.

- Are there features in the history that suggest the diagnosis? Accurate history taking alone is a key stage and often leads to the diagnosis or may suggest the strategy of evaluation.

- Is heart disease present or absent? The absence of signs of suspected or overt heart disease virtually excludes a cardiac cause of syncope with the exception of syncope accompanied by palpitations which could be due to paroxysmal tachycardia (especially paroxysmal supraventricular tachycardia). Conversely, the presence of heart disease at the initial evaluation is a strong predictor of a cardiac cause of syncope, but its specificity is low as about half of patients with heart disease have a non-cardiac cause of syncope.

Table 1 provides the clinical classification of the principal known causes of syncope proposed by the Task Force on Syncope of the ESC. ${ }^{2}$ The subdivision of syncope is based on pathophysiology as follows:

- "Neurally-mediated (reflex) syncope" refers to a reflex response that, when triggered, gives rise to vasodilatation and/or bradycardia; however the contribution of each of these two factors to systemic hypotension and cerebral hypoperfusion may differ considerably. The triggering events might vary considerably in individual patients. The "classical vasovagal syncope" is mediated by emotional or orthostatic stress and can be diagnosed by history taking. "Carotid sinus syncope" is defined as syncope which, by history, seems to occur in close relationship to accidental mechanical manipulation of the carotid sinuses, and which can be reproduced by carotid sinus massage. "Situational syncope" refers to those forms of neurally-mediated syncope associated with specific scenarios (for example, micturition, coughing, defaecating, etc). Often, however, neurallymediated reflex syncopes have "non-classical" presentations. These forms are diagnosed by minor clinical criteria, exclusion of other causes for syncope (absence of structural heart disease) and positive response to tilt testing or carotid sinus massage. Examples of non-classical vasovagal syncope include episodes without clear triggering events or premonitory symptoms.

- "Orthostatic hypotension" refers to syncope in which the upright position (most often the movement from sitting or lying to an upright position) causes arterial hypotension. This occurs when the autonomic nervous system is incapacitated and fails to respond to the challenges imposed by upright position. A second major cause is "volume depletion" in which the autonomic nervous system is itself not deranged, but is unable to maintain blood pressure due to decreased circulating volume. Note that vasovagal syncope can also be provoked by standing (for example, soldiers fainting on parade), but these events are grouped under "neurally-mediated (reflex) syncope".

- "Cardiac arrhythmias" can cause a decrease in cardiac output, which usually occurs irrespective of circulatory demands.

- "Structural heart disease" can cause syncope when circulatory demands outweigh the impaired ability of the heart to increase its output. 


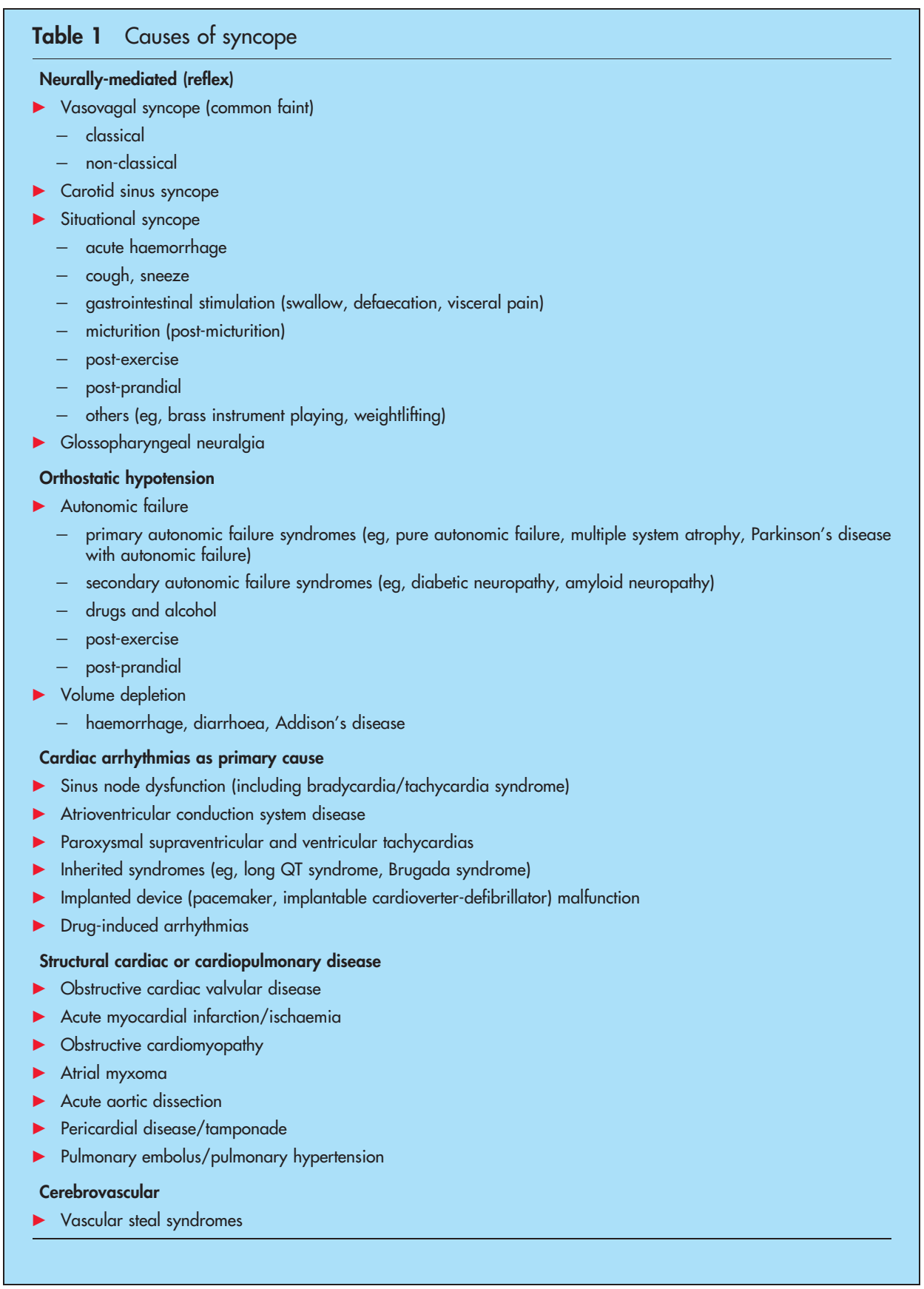

- "Steal" syndromes are rare, but can cause syncope when blood supply is diverted from the brain to another organ (the most common example is the so-called "subclavian steal syndrome" $e^{\prime \prime}$.

Table 2 lists the most common conditions misdiagnosed as causes of syncope. Such a differentiation is crucial because the clinician is usually confronted with patients in whom sudden loss of consciousness has been provoked by causes not associated with decreased cerebral blood flow such as seizure and/or conversion reaction. Several disorders may resemble syncope in two different ways. In some, consciousness is truly lost, but the mechanism is not related to cerebral hypoperfusion: epilepsy, metabolic disorders (including hypoxia and hypoglycaemia) and intoxications. In several other disorders, consciousness is only apparently lost; this is the case in "psychogenic pseudo-syncope", cataplexy and drop attacks.

The initial evaluation may lead to certain or suspected diagnosis or no diagnosis (here termed as unexplained syncope) (fig 1).

\section{CERTAIN DIAGNOSIS}

Initial evaluation may lead to a certain diagnosis based on symptoms, physical signs, or ECG findings. Under such circumstances, no further evaluation may be needed and treatment, if any, can be planned. The results of the initial evaluation are most often diagnostic of the cause of syncope in the following situations: 
Table 2 Causes of non-syncopal attacks (commonly misdiagnosed as syncope)

Disorders without any impairment of consciousness

- Falls

- Cataplexy

- Drop attacks

- Psychogenic pseudo-syncope

- Transient ischaemic attacks (TIA) of carotid origin

Disorders with partial or complete loss of consciousness

- Metabolic disorders, including hypoglycaemia, hypoxia, hyperventilation with hypocapnia

- Epilepsy

- Intoxications

- Vertebro-basilar TIA

- Classical vasovagal syncope is diagnosed if precipitating events such as fear, severe pain, emotional distress, instrumentation or prolonged standing are associated with typical prodromal symptoms.

- Situational syncope is diagnosed if syncope occurs during or immediately after urination, defaecation, cough or swallowing.

- Orthostatic syncope is diagnosed when there is documentation of orthostatic hypotension (defined as a decrease in systolic blood pressure $\geqslant 20 \mathrm{~mm} \mathrm{Hg}$ or a decrease of systolic blood pressure to $<90 \mathrm{~mm} \mathrm{Hg}$ ) associated with syncope or presyncope.
- Cardiac ischaemia-related syncope is diagnosed when symptoms are present with ECG evidence of acute ischaemia with or without myocardial infarction. However, in this case further determination of the specific ischaemia-induced aetiology may be necessary (for example, neurally-mediated hypotension, tachyarrhythmia, ischaemia-induced atrioventricular block, etc).

- Arrhythmia-related syncope is diagnosed by ECG when there is:

- sinus bradycardia $<40$ beats/min or repetitive sinoatrial blocks or sinus pauses $>3 \mathrm{~s}$ in the absence of medications known to have negative chronotropic effect

- second degree Mobitz II or third degree atrioventricular block

- alternating left and right bundle branch block

- rapid paroxysmal supraventricular tachycardia or ventricular tachycardia

- pacemaker malfunction with cardiac pauses

However, it is important to bear in mind that syncope is often multifactorial. The latter is especially true in older individuals. Thus, careful consideration should be given to multiple potential interacting factors (for example, diuretics in older patients already susceptible to orthostatic hypotension, myocardial ischaemia in the setting of moderate aortic stenosis, etc).

\section{SUSPECTED DIAGNOSIS}

Commonly, the initial evaluation leads to a suspected diagnosis, when one or more of the features listed in tables 3 and 4 are present, which needs to be confirmed by tests (table 5 ).

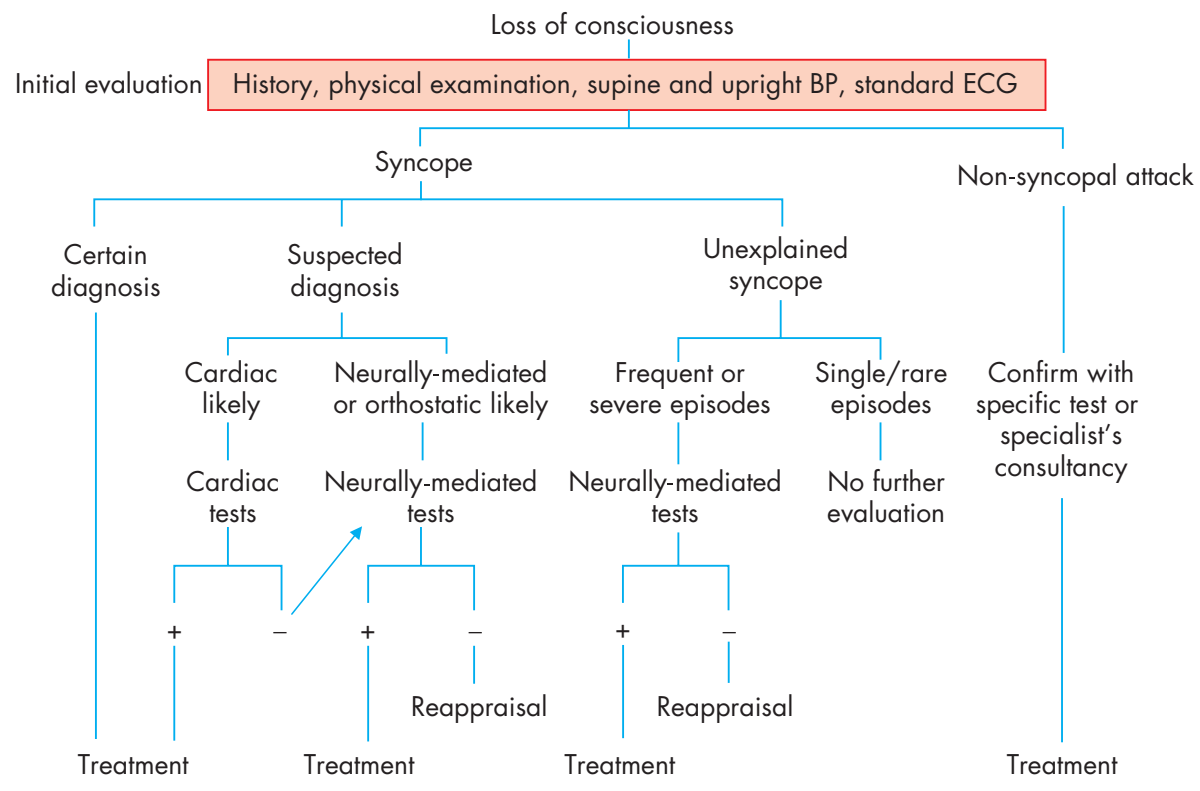

Figure 1 Flow diagram outlining an approach to the evaluation of loss of consciousness based on the initial evaluation, proposed by the Task Force on Syncope of the European Society of Cardiology' 2. Differentiating true syncope from other "non-syncopal" conditions associated with real or apparent transient loss of consciousness is generally the first diagnostic step and influences the subsequent diagnostic strategy. For the classification of syncope refer to table 1 and for the classification of non-syncopal attack refer to table 2. The conditions in which the results of the initial evaluation are diagnostic of the cause of syncope and no further evaluation is required are listed as recommendations in the section "The diagnostic strategy based on the initial evaluation". The features which suggest a cardiac or a neurally-mediated cause of syncope are listed in tables 3 and 4 . Among cardiac investigations, echocardiography, prolonged electrocardiographic monitoring, stress test, electrophysiological study and implantable loop recorder are most useful. Among neurally-mediated investigations, tilt test, carotid sinus massage and implantable loop recorder are most useful. When a cardiac diagnosis cannot be confirmed, neurally-mediated tests are usually performed. Once the evaluation, as outlined, is completed and no cause of syncope is determined, reappraisal of the work-up may be needed. BP, blood pressure; ECG, electrocardiogram. 


Table 3 Clinical features suggestive of specific causes of
syncope
Neurally-mediated syncope
Absence of cardiac disease
Long history of syncope
After sudden unexpected unpleasant sight, sound, smell or pain
Prolonged standing or crowded, hot places
Nausea, vomiting associated with syncope
During or in the absorptive state after a meal
With head rotation, pressure on carotid sinus (as in tumours, shaving,
tight collars)
After exertion
Syncope caused by orthostatic hypotension
After standing up
Temporal relationship with start of medication leading to hypotension or
changes of dosage
Prolonged standing especially in crowded, hot places
Presence of autonomic neuropathy or parkinsonism
Carder exertion
Cardiac syncope
Presence of severe structural heart disease
D During exertion, or supine
Preceded by palpitation or accompanily history of sudden death

The presence of suspected or certain heart disease is associated with a higher risk of arrhythmias and mortality at one year. In these patients, cardiac evaluation (echocardiography, stress testing, electrophysiological study and prolonged ECG monitoring including loop recorder) is recommended.

If cardiac evaluation does not show evidence of arrhythmia as a cause of syncope, evaluation for neurally-mediated syndromes is recommended only in those with recurrent or severe syncope. It includes tilt testing, carotid sinus massage, and ECG monitoring, and often further requires implantation of an implantable loop recorder (ILR). The majority of patients with single or rare episodes in this setting have a high likelihood of neurally-mediated syncope and tests for confirmation are usually not necessary.

Neurologic disease may cause transient loss of consciousness (for example, certain seizures), but is almost never the cause of syncope. Thus, neurologic testing may be needed to distinguish seizures from syncope in some patients, but these should not be considered as essential elements in the evaluation of the basis of true syncope. The possible contribution of electroencephalography (EEG), computed tomography and magnetic resonance imaging of the brain is to disclose abnormalities caused by epilepsy; there are no specific EEG findings for any loss of consciousness other than epilepsy. Accordingly, several studies conclusively showed that EEG monitoring was of little use in unselected patients with syncope. Thus, EEG is not recommended for patients in whom syncope is the most likely cause for a transient loss of consciousness. Carotid TIAs are not accompanied by loss of consciousness. Therefore, carotid

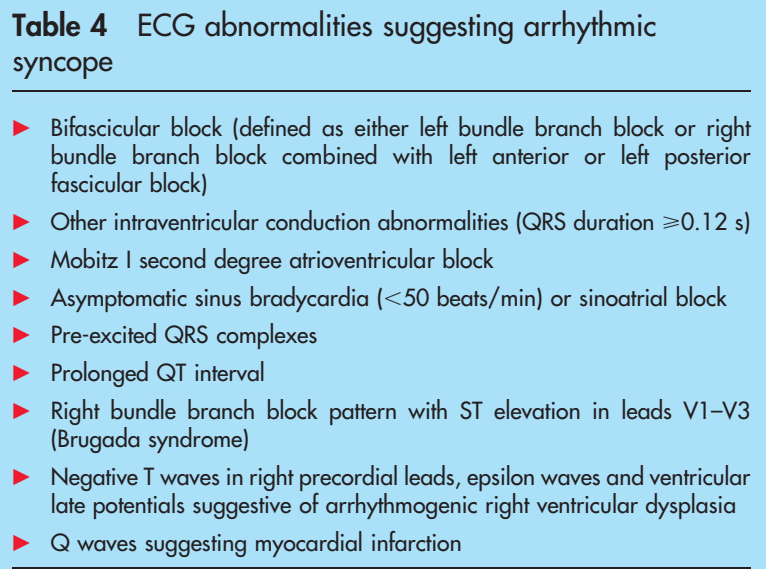

- Bifascicular block (defined as either left bundle branch block or right bundle branch block combined with left anterior or left posterior fascicular block)

- Other intraventricular conduction abnormalities (QRS duration $\geqslant 0.12 \mathrm{~s}$ )

- Mobitz I second degree atrioventricular block

- Asymptomatic sinus bradycardia $(<50$ beats $/ \mathrm{min})$ or sinoatrial block

- Pre-excited QRS complexes

- Prolonged QT interval

- Right bundle branch block pattern with ST elevation in leads V1-V3 (Brugada syndrome)

- Negative T waves in right precordial leads, epsilon waves and ventricular late potentials suggestive of arrhythmogenic right ventricular dysplasia

- $Q$ waves suggesting myocardial infarction

Doppler ultrasonography is not required in patients with syncope. ${ }^{4}$

If the diagnosis is confirmed, treatment may be initiated; if not, a reappraisal process may be useful.

\section{UNKNOWN DIAGNOSIS}

The cause of syncope may remain unexplained after the initial evaluation. The strategy varies according to the severity and frequency of the episodes (fig 1). For patients with unexplained syncope the most likely diagnosis is neurally-mediated for which the appropriate tests are described above. The majority of patients with single or rare episodes in this category probably have neurally-mediated syncope and tests for confirmation are usually not necessary.

\section{REAPPRAISAL}

Once the evaluation is completed and the cause of syncope is undetermined, reappraisal of the work-up is needed since subtle findings or new historical information may change the strategy. Reappraisal may consist of obtaining additional details of history and re-examining the patient, placement of an ILR if not previously undertaken, as well as review of the entire workup. If new clues to possible cardiac or neurological disease are yielded, further cardiac and neurological assessment are recommended. In these circumstances, consultation with appropriate specialists may be useful. Psychiatric assessment is recommended in patients with frequent recurrent syncope who have many other somatic complaints and initial evaluation raises concerns about stress, anxiety and possible other psychiatric disorders.

\section{DIAGNOSTIC YIELD AND PREVALENCE OF CAUSES OF SYNCOPE}

The EGSYS study ${ }^{5}$ was a prospective systematic evaluationbased on strict adherence to the Guidelines on Syncope of the ESC-of consecutive patients referred for syncope to the emergency departments of several general hospitals. The EGSYS study was aimed at assessing the management of syncope as recently defined by ESC guidelines. ${ }^{23}$ The results of this study probably assess the current standard for the management of syncope. A definite diagnosis was established in $98 \%$ (unexplained in 2\%): neurally-mediated syncope accounted for $66 \%$ of diagnoses, orthostatic hypotension $10 \%$, 
Table 5 Most useful and less useful tests

\begin{tabular}{lll}
\hline & Test & Suspected diagnosis \\
\hline Most useful & Carotid sinus massage & Neurally-mediated \\
& Tilt testing & Neurally-mediated \\
& Echocardiogram & Cardiac \\
& Holter/external loop monitoring & Cardiac \\
& Electrophysiological test & Cardiac \\
& Exercise stress testing & Cardiac \\
& Implantable loop recorder & Neurally-mediated and cardiac \\
Less useful (indicated & Electroencephalography & Epilepsy and TIA \\
only in selected cases) & Brain computed tomography & Epilepsy and TIA \\
& Brain magnetic resonance imaging & Epilepsy and TIA \\
& Carotid Doppler sonography & Epilepsy and TIA \\
& Coronary angiography & Cardiac \\
& Pulmonary computed tomography/scintigraphy & Cardiac \\
& Chest $x$-ray & Cardiac \\
& Abdominal ultrasound examination & Co-morbidities \\
\hline \multirow{2}{*}{ TIA, transient cerebral ischaemic attack. } & \\
\hline
\end{tabular}

primary arrhythmias $11 \%$, structural cardiac or cardiopulmonary disease $5 \%$, and non-syncopal attacks 6\% (fig 2). The initial evaluation established a diagnosis in 50\% of cases. Hospitalisation for the management of syncope was appropriate in $25 \%$ and was required for other reasons in a further $13 \%$ of cases. The median in-hospital stay was 5.5 days (interquartile range 3-9 days). Apart from the initial evaluation, a mean (SD) of 1.9 (1.1) appropriate tests per patient were performed in 193 patients and led to a final diagnosis in 182 of these (94\%). This study showed that a guideline-based standardised method of syncope management improved the overall diagnostic yield and reduced hospital admissions, resource consumption and overall costs compared to previous population-based studies. ${ }^{6-10}$

\section{TREATMENT}

Need for mechanism-guided specific treatment

A major issue in the use of diagnostic tests is that syncope is a transient symptom and not a disease. Typically patients are asymptomatic at the time of evaluation and the opportunity to capture a spontaneous event during diagnostic testing is rare. This type of reasoning leads, of necessity, to uncertainty in establishing a cause. Establishing the basis for syncope (that is, determining the "diagnosis") is a prerequisite for advising patients with regard to prognosis, and to developing an effective treatment strategy. However, arriving at the diagnosis can be difficult, and is often marked by the undertaking of costly and often useless diagnostic procedures. The ultimate goal of diagnostic testing is to establish a sufficiently strong correlation between syncope and detected abnormalities to permit both an assessment of prognosis and initiation of an appropriate treatment plan. Knowledge of what occurs during a spontaneous syncopal episode is ideally the gold standard for syncope evaluation. For this reason it is likely that ILRs will become increasingly important in the assessment of the syncope patient, and their use will increasingly be appropriate instead of, or before, many current conventional investigations. This early ILR approach implies the need for a careful initial risk stratification in order to exclude from such a strategy patients with potential life threatening conditions.

\section{Neurally-mediated (reflex) syncope}

Patients who seek medical advice after having experienced a vasovagal faint require reassurance and education regarding the nature of the disease and the avoidance of triggering events. In general, education and reassurance are sufficient for most patients. Modification or discontinuation of hypotensive drug

\section{Causes of loss of consciousness}
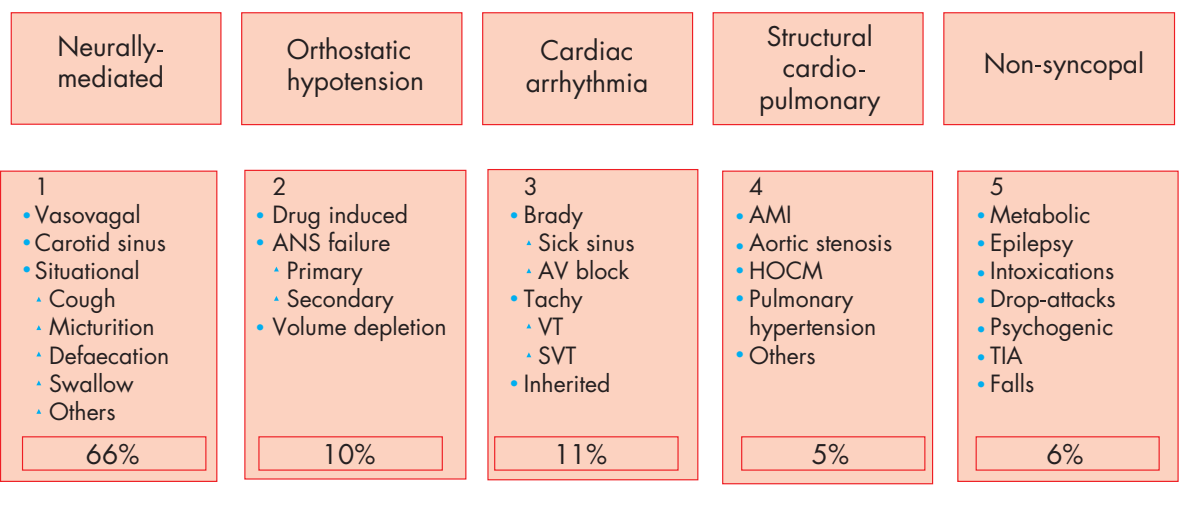

\begin{tabular}{l}
\hline 5 \\
- Metabolic \\
- Epilepsy \\
- Intoxications \\
- Drop-attacks \\
- Psychogenic \\
- TIA \\
- Falls \\
\hline $6 \%$ \\
\hline
\end{tabular}

Unknown cause $=2 \%$
Figure 2 Prevalence of the causes of syncope in the EGSYS-2 study. ${ }^{5}$ The EGSYS study was aimed at assessing the management of syncope as recently defined by ESC guidelines. ${ }^{2}$ The results of this study probably represent the current standard for the management of syncope. AMI, acute myocardial infarction; ANS, autonomic nervous system; AV, atrioventricular; HOCM, hypertrophic obstructive cardiomyopathy; SVT, supraventricular tachyarrhythmia; TIA, transient ischaemic attack; VT, ventricular tachycardia. 
treatment for concomitant conditions and avoidance of triggering situations are other first line measures for the prevention of syncope recurrences. Treatment is not necessary for patients who have sustained a single syncope and are not having syncope in a high risk setting. ${ }^{12}$

Additional treatment may be necessary in high risk or high frequency settings when:

- syncope is very frequent-for example, alters the quality of life

- syncope is recurrent and unpredictable (absence of premonitory symptoms) and exposes patients to "high risk" of trauma

- syncope occurs during the prosecution of a "high risk" activity (for example, driving, machine operation, flying, competitive athletics, etc).

Non-pharmacological "physical" treatments are emerging as a new front line treatment of vasovagal syncope. In highly motivated patients with recurrent vasovagal symptoms, the prescription of progressively prolonged periods of enforced upright posture (so-called "tilt-training") may reduce syncope recurrence. However, this treatment is hampered by the low compliance of the patients in continuing the training programme for a long period. ${ }^{11}$ Two recent clinical trials ${ }^{12}{ }^{13}$ have shown that isometric counterpressure manoeuvres of the legs (leg crossing), or of the arms (hand grip and arm tensing), are able to induce a significant blood pressure increase during the phase of impending vasovagal syncope, which allow the patient to avoid or delay losing consciousness in most cases.

Many drugs have been used in the treatment of vasovagal syncope ( $\beta$-blockers, disopyramide, scopolamine, clonidine, theophylline, fludrocortisone, ephedrine, etilefrine, midodrine, clonidine, serotonin reuptake inhibitors, etc). In general, while the results have been satisfactory in uncontrolled trials or short-term controlled trials, long-term placebo-controlled prospective trials have failed to show any benefit of the active drug over placebo. $\beta$-adrenergic blocking drugs have failed to be effective in five of six long-term follow-up controlled studies. ${ }^{14-}$ 19 Vasoconstrictor drugs are potentially more effective in orthostatic hypotension caused by autonomic dysfunction than in neurally-mediated syncope. Etilefrine proved to be ineffective. ${ }^{20}$ To date there are insufficient data to support the use of any other pharmacological treatment for vasovagal syncope.

The role of cardiac pacing for vasovagal syncope is not yet established. In unselected tilt-positive patients, pacing for vasovagal syncope has produced controversial results. Pooling together the results of five randomised controlled trials ${ }^{21-25}$ syncope recurred in $21 \%$ of the paced patients and in $44 \%$ of un-paced patients. Specifically, the efficacy of pacemaker therapy was questioned after two recent controlled trials failed to prove superiority of cardiac pacing over placebo of unselected patients with positive tilt testing. ${ }^{24} 25$ It seems that pacing therapy might be effective in some but not in all patients. This is not surprising if we consider that pacing is probably efficacious for asystolic reflex but has no role in combatting hypotension which is frequently the dominant reflex in neurally-mediated syncope. A recent study using the ILR as reference standard ${ }^{26}$ showed that only about half of the patients had an asystolic pause recorded at the time of spontaneous syncope. A new strategy of delaying treatment until ILR documentation of the mechanism of syncope with cardiac
Diagnosis and treatment of syncope: key points

- The starting point for evaluation of syncope is the "initial evaluation" that consists of history taking and physical examination, including orthostatic blood pressure measurements and standard electrocardiogram

- The initial evaluation may lead to certain diagnosis, or suspected diagnosis that needs to be confirmed by appropriate diagnostic tests, or no diagnosis

- The strategy of evaluation varies according to the severity and frequency of the episodes and the presence or absence of heart disease

- In general, the absence of suspected or certain heart disease excludes a cardiac cause of syncope

- Conversely, the presence of heart disease is a strong predictor of a cardiac cause of syncope and virtually includes all cardiac syncopes, but its specificity is low as about half of patients with heart disease have a non-cardiac cause of syncope.

- Determining the mechanism of syncope is a prerequisite for advising patients with regard to prognosis, and to developing an effective mechanism-specific treatment

- Most patients with syncope require only reassurance and education regarding the nature of the disease and the avoidance of triggering events

pacing, limited to those patients who had asystole at the time of syncope, proved to be very effective by reducing syncopal recurrence rate of $90 \%$ to an absolute value of $5 \%$ at one year. ${ }^{27}$ It must be underlined that the decision to implant a pacemaker needs to be kept in the clinical context of a benign condition which frequently affects young patients. Thus, cardiac pacing should be limited as a last resort choice to a very selected small proportion of patients affected by severe vasovagal syncope.

Cardiac pacing appears to be beneficial in the carotid sinus syndrome and, although only one relatively small randomised controlled trial has been undertaken, pacing is acknowledged to be the treatment of choice when bradycardia has been documented. ${ }^{12}$ Single chamber atrial pacing is not appropriate for vasovagal syncope, and dual-chamber pacing is generally preferred over single chamber ventricular pacing.

\section{Orthostatic hypotension}

Drug-induced autonomic failure is probably the most frequent cause of orthostatic hypotension. The principal treatment strategy is elimination of the offending agents, mainly diuretics and vasodilators. Alcohol is also commonly associated with orthostatic intolerance. ${ }^{12}$

Additional treatment principles, used alone or in combination, are appropriate for consideration on an individual patient basis $^{12}$ :

- chronic expansion of intravascular volume by encouraging a higher than normal salt intake and fluid intake of 2-2.5 litres per day.

- fludrocortisone in low dose (0.1-0.2 mg per day)

- raising the head of the bed on blocks to permit gravitational exposure during sleep

- reduce vascular volume into which gravitation induced pooling occurs by use of abdominal binders and/or waist height support stockings or garments 
- introduce physical counter manoeuvres such as leg crossing, gripping or squatting

- use of drugs which increase peripheral resistance (midodrine 5-15 mg three times daily).

\section{Cardiac arrhythmias as primary cause}

Syncope caused by cardiac arrhythmias must receive treatment appropriate to the cause in all patients in whom it is lifethreatening and when there is a high risk of injury.

Treatment may be warranted when:

- the culprit arrhythmia has not been demonstrated conclusively, but a diagnosis of life-threatening arrhythmia is presumed from surrogate data

- the culprit arrhythmia has been identified but is not lifethreatening or presenting a high risk of injury.

Cardiac pacing, implantable cardioverter-defibrillators, and catheter ablation are the usual treatments of syncope caused by cardiac arrhythmias, depending on the mechanism of syncope.

\section{Structural cardiac or cardiopulmonary disease}

Treatment is best directed at amelioration of the specific structural lesion or its consequences.

In compliance with EBAC/EACCME guidelines, all authors participating in Education in Heart have disclosed potential conflicts of interest that might cause a bias in the article

\section{REFERENCES}

1 Brignole M, Alboni P, Benditt D, et al. Guidelines on management (diagnosis and treatment) of syncope-Update 2004. Europace 2004;6:467-537.

2 Brignole M, Alboni P, Benditt D, et al. Guidelines on management (diagnosis and treatment) of syncope-Update 2004-Executive summary and recommendations. Eur Heart J 2004;25:2054-72.

- References 1 and 2 are important consensus documents describing a standardised strategy for the evaluation and the treatment of patients with syncope

3 Alboni $\mathrm{P}$, Brignole $M$, Menozzi $C$, et al. The diagnostic value of history in patients with syncope with or without heart disease. J Am Coll Cardiol 2001;37:1921-8.

4 Linzer M, Yang E, Estes M, et al. Diagnosing syncope. Part II: unexplained syncope. Ann Intern Med, 1997; 127, 76-86.

5 Brignole M, Menozzi C, Bartoletti A, et al. A new management of syncope. Prospective systematic guideline-based evaluation of patients referred urgently to general hospitals. Eur Heart J 2006;27:78-82.

6 Ammirati F, Colivicchi F, Minardi G, et al. Hospital management of syncope: the OESIL study. G Ital Cardiol 1999;29:533-9.

7 Disertori M, Brignole M, Menozzi C, et al. Management of syncope referred for emergency to general hospitals. Europace 2003;5:283-91.

8 Farwell DJ, Sulke AN. Does the use of a syncope diagnostic protocol improve the investigation and management of syncope? Heart 2004;90:52-8.

9 Blanc JJ, L'Her C, Touiza A, et al. Prospective evaluation and outcome of patients admitted for syncope over 1 year period. Eur Heart J 2002;23:815-20.

10 Ammirati F, Colivicchi F, Santini M. Diagnosing syncope in the clinical practice. Implementation of a simplified diagnostic algorithm in a multicentre prospective trial-the OESIL 2 study (Osservatorio Epidemiologico della Sincope nel Lazio). Eur Heart J 2000;21:935-40.

11 Ector H, Reybrouck T, Heidbuchel H, et al. Tilt training: a new treatment for recurrent neurocardiogenic syncope or severe orthostatic intolerance. Pacing Clin Electrophysiol 1998;21:193-6.
- The first description of a new therapy for vasovagal syncope-tilt training.

12 Brignole $M$, Croci $F$, Menozzi $C$, et al. Isometric arm counter-pressure maneuvers to abort impending vasovagal syncope. J Am Coll Cardiol 2002;40:2054-60.

13 Krediet $P$, van Dijk N, Linzer M, et al. Management of vasovagal syncope: controlling or aborting faints by leg crossing and muscle tensing. Circulation 2002;106:1684-9.

- The first description of another new therapy for vasovagal syncopephysical manoeuvres.

14 Brignole M, Menozzi C, Gianfranchi L, et al. A controlled trial of acute and longterm medical therapy in tilt-induced neurally mediated syncope. Am J Cardiol 1992;70:339-42.

15 Sheldon R, Rose S, Flanagan $P$, et al. Effects of beta blockers on the time to first syncope recurrence in patients after a positive isoproterenol tilt table test. Am J Cardiol 1996;78:536-9.

16 Di Gerolamo E, Di lorio C, Sabatini P, et al. Effects of different treatments vs no treatment on neurocardiogenic syncope. Cardiologia 1998:43:833-7.

17 Flevari P, Livanis E, Theodorakis G, et al. Vasovagal syncope: a prospective, randomized, cross-over evaluation of the effects of propranolol, nadolol and placebo on syncope recurrence and patients' well-being. J Am Coll Cardiol 2002;40:499-504.

18 Madrid A, Ortega I, Rebollo GJ, et al. Lack of efficacy of atenolol for the prevention of neurally-mediated syncope in highly symptomatic population: a prospective double-blind, randomized and placebo-controlled study. J Am Coll Cardiol 2001;37:554-7.

- Double-blind placebo-controlled trial showing no benefit of $\beta$-blocker treatment for vasovagal syncope.

19 Ventura R, Maas R, Zeidler D, et al. A randomized and controlled pilot trial of $\beta$ blockers for the treatment of recurrent syncope in patients with a positive or negative response to head-up tilt test. Pacing Clin Electrophysiol 2002;25:816-21.

20 Raviele A, Brignole M, Sutton $R$, et al. Effect of etilefrine in preventing syncopal recurrence in patients with vasovagal syncope: a double-blind, randomized, placebo-controlled trial. The Vasovagal Syncope International Study. Circulation 1999;99: 1452-7.

- Double-blind placebo-controlled trial showing no benefit of $\alpha$-agonist drug treatment for vasovagal syncope.

21 Sutton R, Brignole M, Menozzi C, et al. Dual-chamber pacing in treatment of neurally-mediated tilt-positive cardioinhibitory syncope. Pacemaker versus no therapy: a multicentre randomized study. Circulation 2000;102:294-9.

22 Connolly SJ, Sheldon R, Roberts RS, et al. Vasovagal pacemaker study investigators. The North American vasovagal pacemaker study (VPS): a randomized trial of permanent cardiac pacing for the prevention of vasovagal syncope, J Am Coll Cardiol 1999:33:16-20.

23 Ammirati $F$, Colivicchi $F$, Santini $M$, et al. Permanent cardiac pacing versus medical treatment for the prevention of recurrent vasovagal syncope. A multicenter, randomized, controlled trial. Circulation 2001;104:52-7.

24 Connolly SJ, Sheldon R, Thorpe KE, et al, for the VPS II Investigators. Pacemaker therapy for prevention of syncope in patients with recurrent severe vasovagal syncope: Second Vasovagal Pacemaker Study (VPS II). JAMA 2003;289:2224-9.

- Double-blind placebo-controlled trial showing no benefit of cardiac pacing for tilt-positive unselected vasovagal syncope patients.

25 Raviele A, Giada F, Menozzi C, et al. A randomized, double blind placebocontrolled study of permanent cardiac pacing for the treatment of recurrent tiltinduced vasovagal syncope. The vasovagal syncope and pacing trial (SYNPACE). Eur Heart J 2004;25:1541-8.

26 Moya A, Brignole M, Menozzi C, et al. Mechanism of syncope in patients with isolated syncope and in patients with tilt-positive syncope. Circulation $2001 ; 104: 1261-7$

- This study, using an implantable loop recorder, showed that half of the patients with tilt-positive vasovagal syncope have a long asystolic pause at the time of the spontaneous syncope and that there is no difference between these patients and those with tilt-negative isolated syncope.

27 Brignole M, Sutton R, Menozzi C, et al. Early application of an implantable loop recorder allows effective specific therapy in patients with recurrent suspected neurally-mediated syncope. Eur Heart J 2006;27:1085-92.

- This study showed that a strategy of early implantation of an implantable loop recorder and specific therapy delayed until documentation of the mechanism of syncope is safe and very effective. 